\title{
EDUCACIÓN MUSICAL PARA EL DESARROLLO SOSTENIBLE: UNA REVISIÓN DOCUMENTAL
}

\author{
Educação musical para o desenvolvimento \\ sustentável: uma revisão de literatura
}

\author{
Music education for the sustainable \\ development: a documental review
}

\author{
OSWALDO ANTONIO RODRÍGEZ REINOSO \\ Fundación Parindé Centro de Investigación y Documentación Conservatorio "Vicente Emilio Sojo" \\ oarodriguezr@hotmail.com \\ JOSEMANUEL LUNA-NEMECIO \\ Centro Universitario CIFE \\ josemanuelluna@cife.edu.mx
}

\begin{abstract}
Resumen: El presente avance de investigación muestra la construcción de un registro y análisis documental tendiente a explorar perspectivas onto-epistémicas y metodológicas que permitan articular categorías como educación musical, desarrollo sostenible y educación ambiental para el planteamiento de una educación musical desde la práctica colectiva y orientada al desarrollo sostenible. Desde un enfoque cualitativo se aplicó la metodología de análisis documental para realizar la consulta directa de artículos científicos indizados en bases de datos tomando en consideración criterios de búsqueda y selección. Los resultados obtenidos develan la pertinencia de las categorias de análisis conjugadas con lo cual se abren los espacios para considerar una propuesta de educación musical como praxis educativa orientada al desarrollo sostenible.
\end{abstract}

Palabras clave: Educación musical. Desarrollo sostenible. Educación ambiental. Socioformación.

Resumo: A presente pesquisa mostra a construção de um registro e análise documental tendendo a explorar perspectivas onto-epistemológicas e metodológicas que permitem articular categorias como educação musical, desenvolvimento sustentável e educação ambiental para a abordagem de uma educação musical a partir da prática coletiva até o desenvolvimento sustentável. A partir de uma abordagem qualitativa foi aplicada a metodologia de análise documental para realizar consulta direta de artigos científicos indexados em bases de dados, levando em consideração critérios de busca e seleção. Os resultados obtidos revelam a relevância das categorias de análise combinadas de tal forma a abrir espaços para considerar uma proposta de educação musical como prática educativa orientada para o desenvolvimento sustentável.

Palavras-chave: educação musical, desenvolvimento sustentável, educação ambiental, sócio formação

\begin{abstract}
The present advance of research shows the construction of a register and documentary analysis tending to explore onto-epistemic and methodological perspectives that allow articulating categories as musical education, sustainable development and environmental education for the approach of a musical education from the collective practice and oriented to the sustainable development. From a qualitative approach, we proceeded through the direct consultation of scientific articles indexed in databases taking into consideration search and selection criteria. The results obtained reveal the pertinence of the categories of conjugated analysis with which the spaces are opened to consider a musical education proposal as an educational praxis oriented to sustainable social development.
\end{abstract}

Keywords: Music education. Sustainable social development. Environmental education. Socioformation. 


\section{PROBLEMA}

Acudimos en los últimos años a la re-significación del concepto de ambiente (Aguirre, 2017; Torres, 2015; Molina-Motos, 2019) y, con ello, el de educación ambiental (Galindo, 2015; Valderrama-Hernández; Alcántara; Limón 2017; Mejía, 2016), comúnmente comprendida como el conjunto de conocimientos relacionados con la preservación de la naturaleza a partir de diversos enfoques pedagógicos-didácticos que rondan el entorno físico y humano, lo mismo que la promoción sobre el cuidado y respeto al ambiente (Miedijensky; Abramovich, 2019, p. 2). La educación ambiental (LunaNemecio, 2019a; Flores-Mondragón, 2019) tiene por reto "educar para cambiar la sociedad, que se asuman actitudes de pensamiento hacia un desarrollo humano en consonancia con su compromiso planetario" (González-Escobar, 2017, p. 6).

Por su parte, la sostenibilidad en tanto corriente de pensamiento, se levanta como alternativa para orientar procesos que conlleven a la redefinición de conceptos, generación de nuevas aproximaciones desde lo teórico y metodológico a partir de la crisis ambiental que signa la modernidad continuada en la que nos encontramos (Luna-Nemecio; Tobón; Juárez-Hernández, 2019). La sostenibilidad implica la condición finita de los recursos de la tierra, la producción limpia, el crecimiento exponencial de la población y la contaminación (Zarta, 2018, p. 412). Importante es por igual reconocer la diferenciación del concepto de desarrollo social sostenible (Luna-Nemecio; Tobón; Juárez-Hernández, 2019) ligado al crecimiento económico de los estados naciones, la acumulación de capital y el desarrollo tecnológico, ya que este criterio, propio de la tesis capitalista (LunaNemecio, 2018), no vincula la dimensión social y cultural, lo cual juega un papel determinante en el ejercicio de una ética que ponga como criterio la preservación de la vida. Así, la sostenibilidad como criterio integrador impulsa a la contextualización y problematización a través de las prácticas educativas (Santoyo-Ledesma, 2019) con el propósito de crear una recurrente acción crítico reflexiva vinculada a los retos socio-ambientales y al fortalecimiento de valores colectivos (Jacobi; Ferraz de Toledo; Grandisoli, 2016, p. 2).

De igual modo, si se considera que la noción de ambiente no sólo implica el mundo físico-natural, sino el entorno en el que la relación intersubjetiva se propicia, donde se ejerce la acción política del relacionamiento humano entre sí, se puede por tanto perfilar una reflexión sobre prácticas educativas que se orienten y enmarquen en la noción de sostenibilidad, entendiendo por esto, un proceso tendiente a propiciar condiciones que fortalezcan y orienten las dimensiones sociales, culturales, políticas, económicas y ambientales necesarias para la preservación y aumento de la vida. 
La socioformación (Tobón, 2015), como alternativa latinoamericana para la transformación de los procesos formativos, o lo que es igual, el cambio paradigmático en la concepción de la educación por objetivos y contenidos a una educación por competencias cuyo énfasis se hace en la contextualización de los problemas, emerge a partir de la práctica misma, desde el accionar en situación, donde los actores sociales responsables del hecho formativo se encuentran ante la compleja realidad de educar en la era del conocimiento. El proceso de cambio que implica la socioformación hunde sus raíces en la idea de un aprendizaje colaborativo, en comunidad, donde se vive la práctica del colectivo. Esto conlleva a la educación toda a una completa redefinición en todos sus ámbitos, uno de ellos la educación para las artes, en lo específico, la educación musical.

Por su parte, la educación musical (Reis, 2018; García, 2018) no escapa a los problemas estructurales que aqueja la educación como ámbito general del conocimiento. Su pertinencia en los diseños curriculares, la necesidad de fundamentar su permanencia en los planes de estudio, las concepciones pedagógico-didácticas centradas en modelos que no responden a las exigencias de hoy día y su vinculación por medio de prácticas que se orientan al desarrollo social, denotan los momentos de cuestionamientos en sus bases conceptuales, epistemológicas y metodológicas.

En este sentido, la presente investigación explora las posibilidades de articular elementos de orden ontológico y epistemológico para una educación musical orientada desde la socioformación para el desarrollo sostenible (Luna-Nemecio; Tobón; Juárez-Hernández, 2019) a partir de la práctica colectiva de la música sobre la base del enfoque por competencias, siendo los propósitos: 1) analizar las categorías vinculantes al planteamiento central, educación musical, desarrollo sostenible y educación ambiental; 2) formular un corpus ontológico, epistemológico y metodológico a partir del marco categorial propuesto, 3) diseñar el conjunto de competencias y ejes orientadores de una educación musical a partir de la práctica colectiva de la música y 4) consolidar una propuesta curricular de educación musical para el desarrollo sostenible desde la práctica colectiva de la música bajo el enfoque por competencias.

\section{MetodoloGía}

\section{Tipo de estudio}

El planteamiento central de la investigación pasa por la concepción epistémica de la transdisciplinariedad y la complejidad para el abordaje no de un objeto de estudio sino de un campo de problemas (Fernández, 2008, p. 28). La direccionalidad de la investigación encuentra claro dominio en un enfoque cualitativo, entendido como aquel que aborda a profundidad la naturaleza de las realidades desde la consideración de su carácter unitario dinámico (Martínez, 2004 p. 66). El dispositivo metodológico que se plantea 
es la realización de una revisión y registro documental (CIFE, 2016, p. 1) mediante criterios de búsqueda, selección y análisis que permitirán el acercamiento a las categorias fundamentales que orientan la presente investigación.

La revisión y análisis documental "consiste en buscar, seleccionar, organizar y analizar un conjunto de materiales escritos para responderá una o varias preguntas sobre un tema" (Bermeo-Yaffar; Hernández-Mosqueda; Tobón-Tobón, 2016, p. 105). Son aspectos claves en una revisión y análisis documental la pertinencia de la fuente con respecto al problema que se investiga, el establecer categorias de análisis como ejes orientadores, el análisis crítico tendiente a encontrar vacíos en el conocimiento para así proponer soluciones (Ortega-Carbajal; Hernández-Mosqueda; Tobón-Tobón, 2015, p. 143).

\section{Categorías de análisis}

\begin{tabular}{c|c}
\hline Categorias de Análisis & \multicolumn{1}{c}{ Interrogantes de Investigación } \\
\hline Educación musical & $\begin{array}{c}\text { ¿Cómo articular un corpus epistemológico desde la } \\
\text { socioformación y la sostenibilidad para una } \\
\text { educación musical? ¿Cuáles son los elementos que } \\
\text { pueden configurar una educación musical como } \\
\text { praxis para la sostenibilidad? ¿de qué manera puede } \\
\text { Educación ambiental } \\
\text { articularse una educación musical como praxis para } \\
\text { la sustentabilidad dentro de la visión de la educación } \\
\text { formal y no formal? ¿Cuáles son las competencias } \\
\text { necesarias para una educación musical como praxis } \\
\text { para la sostenibilidad? }\end{array}$ \\
\hline
\end{tabular}

Cuadro 1: Categorias de Análisis e Interrogantes de Investigación.

Se procedió consultando fuentes electrónicas y bases de datos tales como: Science Direct, Redalyc, Scopus, Google Académico, Scielo, Latindex, Springeropen en busca de artículos de investigación que tuviesen relación con las categorias propuestas. Se emplearon, para cada caso su correspondencia en el idioma inglés de manera de acceder a fuentes en dicho idioma, siendo las empleadas: music education, sustainable development, environmental education.

\section{Criterios de búsqueda y selección}

Para la determinación de los artículos de investigación rastreados en las fuentes se establecieron los siguientes criterios de selección:

a) Abordar o contener las palabras claves o la pertinencia en relación a los propósitos de la investigación

b) Rango de fecha de la publicación a partir de 2015 a 2019 (vigencia) 
c) Artículo indizado y debidamente identificado

d) Idioma español o inglés

\begin{tabular}{c|c|c|c}
\hline Categoría Consultada & $\begin{array}{c}\text { Tipo de } \\
\text { Documento }\end{array}$ & $\begin{array}{c}\text { Idioma } \\
\text { Español }\end{array}$ & $\begin{array}{c}\text { Idioma } \\
\text { Inglés }\end{array}$ \\
\hline Educación musical & Artículo & 12 & 11 \\
\hline Desarrollo sostenible & Artículo & 16 & 11 \\
\hline Educación ambiental & Artículo & 14 & 11 \\
\hline Competencias docentes & Artículo & 10 & 10 \\
\hline \multicolumn{2}{c|}{ Totales } & $\mathbf{5 2}$ & $\mathbf{4 3}$ \\
\hline
\end{tabular}

Cuadro 2: Detalle de artículos seleccionados por categoría consultada.

\section{ANÁLISIS DE RESULTADOS}

\section{Educación Musical}

El plantearse la tarea de abordar una educación musical para el desarrollo sostenible (Luna-Nemecio; Tobón; Juárez-Hernández, 2019) trae consigo un giro en lo ontológico ${ }^{1}$, epistémico, metodológico y axiológico al situar la acción educativa-musical desde una perspectiva socio-cultural y politica, para vincular la práctica musical en colectivo a la pervivencia de la vida humana, a propiciar espacios de enseñanza-aprendizaje cuyo criterio sea la vida, no sólo en el sentido biológico, sino social, político y cultural. La educación musical, como práctica social, cultural y política, ámbito de educación general y ámbito general de educación (Touriñan; Longueira, 2010), puede orientarse al desarrollo sostenible a partir de una propuesta que contemple en sus bases epistémicas y metodológicas la socioformación, el enfoque por competencias y la transdisciplinariedad.

En una primera instancia y en sentido de lo histórico, se reconocen dos modelos fundamentales en la educación musical, a saber: el modelo jesuita y el modelo conservatorio Schifres; Gonnet (2015). Para el primero de estos modelos, los autores refieren que su acción primaria fue "la sustitución de todo vestigio musical preexistente. Asi se sustituyeron los objetos materiales de la música - específicamente los instrumentos musicales - negando la

\footnotetext{
${ }^{1}$ En lo ontológico, es preciso reconfigurar por una parte los fines de una educación musical conjugando los de orden disciplinar y los de orden socio-cultural, para lo cual la asunción del sujeto colectivo viene a ser el centro de una concepción comunitaria de la práctica musical. El nuevo sujeto no es el virtuoso individual sino la comunidad concertada. Una concepción que para el sistema pedagógico en general debe orientar su praxis en el sentido político-social, en su ethos social (Dussel, 2011), el hombre como centro de la totalidad cultural. La concertación es la reafirmación de la convivialidad que consolida por tanto el diálogo para fortalecer "prácticas tolerantes y de impulso al libre pensamiento que oxigena la práctica educativa" (Varas, 2008: 86).
} 
vinculación entre la música y el entorno vertebrador de las expresiones sonoras de las culturas vernáculas" (p. 55). En lo que concierne al modelo llamado conservatorio, el cual se reconoce a partir de la creación del Conservatorio Nacional de París, se sustenta "sobre la base de alguno de los pilares del modelo jesuita, oportunamente enfatizados y perfeccionados, tales como la alfabetización musical y el desarrollo instrumental preeminente", lo mismo que algunas otras caracteristicas vinculadas a los privilegios en el acceso al aprendizaje musical, al "encuadre diádico y la valoración de los desempeños individuales" (Schifres; Gonnet, 2015, p. 57).

La permanencia de este modelo, anclado principalmente en el desarrollo técnico individual, encuentra en el siglo XX una proliferación importante de corrientes de pensamiento centrados en el hecho pedagógicodidáctico. A principios de ese siglo "se esbozan los primeros intentos de mejorar con la aparición de nuevas metodologías en educación musical" (Sierra, 2018, p. 97-98). El método "Tonic sol-fa" en Inglaterra, el método de Maurice Chevais en Francia son ejemplos representativos. El movimiento de la escuela nueva (Pestalozzi, Decroly, Froebel, Montessori) abrió paso a referentes como Dalcorze, Willems y Martenot y posteriormente a los denominados métodos instrumentales (Orff, Kodaly, Zuzuki). Durante los años 70 y 80, se inicia una "nueva corriente pedagógica musical (G. Self, B. Dennis, J. Paynter o M. Schafer entre otros) con los diferentes tipos de métodos "creativos" donde los niños y niñas empiezan a compartir el ejercicio de la composición musical con los maestros" (p. 97-98).

No encontramos así con una educación musical inserta dentro de la educación formal como un área de conocimiento que comparte espacio junto a las otras áreas del saber general y que responde a una finalidad muy distinta a la educación musical que centra sus esfuerzos en el desarrollo de saberes, conocimientos y destrezas tendientes a la ejecución instrumental, la práctica orquestal y coral propia del modelo conservatorio o de escuelas de música de corte europeo. Desde esta perspectiva, la literatura consultada evidencia la resistencia por parte de la escuela hacia expresiones musicales distintas a las del modelo europeo. A esto se suma, del lado de la educación musical del modelo conservatorio, "la permanencia en modelos educativos anclados en el siglo pasado, en lugar de adaptar las enseñanzas a nuestro contexto socio-cultural" y del lado de la escuela, el hecho de que "los maestros generalistas refieren tener escasa formación musical para poder defender y llevar a cabo prácticas de calidad desde la etapa infantil" (Peñalba, 2017, p. 113).

En este mismo orden de ideas, es también importante destacar "la pérdida de peso curricular de la Educación Musical en todos los niveles educativos y con una dimensión prácticamente internacional es un hecho que nos afecta a todos los profesionales e investigadores del área" (RamírezHurtado, 2017, p. 130). Para Fernández-Jiménez \& Jorquera-Jaramillo (2017) "hay ciertas criticas que apuntan a que parte de esa vulnerabilidad 
de la educación musical se debe a los fallos de la anterior filosofía de la educación musical, basada en el desarrollo del sentido estético" (p. 99).

Otro punto común al que lleva la revisión documental es la importancia de la educación musical en los tiempos actuales. A este respecto, Asprilla (2015) destaca "la importancia de la música para la vida y su permanencia en todas las culturas permiten concebirla como un contexto de desarrollo y aprendizaje, más que como una especialidad profesional" (p. 64). Existe un punto de convergencia desde lo cultural, entendido como proceso formativo y la propia educación institucionalizada, llevando con ello la posibilidad de educar en la música. "El fin último de la educación es formar personas, ciudadanos que puedan vivir en sociedad, personas criticas y sensibles, creativas e imaginativas" (Peñalba, 2017, p. 122).

El aprendizaje en y a través de la música estimula, entre otros aspectos, la confianza en sí mismo, la sensibilidad emocional, competencias sociales, concentración, coordinación motora fina, pensamiento crítico y resolución de problemas (Sicherl; Denac; Konstanca, 2015, p. 95). A su vez, el impacto desde el punto de vista social y la preocupación que dentro del área investigativa tanto educativa como musical ha traído en los últimos años, es uno de los tópicos que a partir de la revisión documental resultan de gran importancia. Ramírez-Hurtado (2017) reseña la aparición en el año de 1992 del primer manual sistemático sobre investigación en el aprendizaje y enseñanza de la música, donde se abordaban temas fundamentales relacionados a las metodologías socioeducativas $y$ temas de orden psicopedagógico, lo mismo que los ámbitos de la educación de la educación musical (p. 133-134). En su análisis a la versión aparecida diez años más tarde encuentra "diferencias sustanciales, aunque podrian pasar inadvertidas en una lectura superficial. La primera de ellas es que la cuestión del impacto social y la política de la EM están presentes de alguna manera en todas las partes del texto" (p. 133-134). El autor comulga con la idea de una "educación musical por la paz" asumiendo el concepto de la transverdad frente a la posverdad, dado el reconocimiento que hace a la educación musical de valorizar ese concepto "concepto ético y epistemológico basado en la persona, comprometido con la sociedad y que es capaz de construir una verdadera comunidad politica, educativa e investigadora" (Ramírez-Hurtado, 2017, p. 145).

Bates (2018) estima necesario acoger la vía de la teoría crítica en la necesidad de "entender la crisis de legitimidad de la educación musical para identificar acciones éticamente sólidas, socialmente justas y ambientalmente sostenibles" (p. 2). Shifres y Gonnet (2015) hacen mención a la acción colonizadora de la educación musical de tradición europea representada tanto en el modelo jesuita y de conservatorio. Los autores plantean una acción decolonial, con la cual afrontar la problemática que se hereda de la educación musical a partir de los modelos ya mencionados. Esto implica, restituir el vínculo vivencia musical y naturaleza, recomponer 
el concepto de colectivo para la práctica musical y la revisión de los enfoques de enseñanza aprendizaje (Shifres; Gonnet, 2015, p. 64-65).

Sobre la noción de lo colectivo como condición de posibilidad de la música, resulta importante añadir el hecho de que "pensar la música en el colectivo, del que todos formamos parte, implica que el destinatario mismo del encuentro musical es el propio colectivo" (Shifres; Gonnet, 2015, p. 65). En este mismo orden de ideas, el concepto de comunidad de prácticas, al vincularse a la música ratifica lo antes desarrollado por los autores citados con respecto al repensar ontologías y epistemologías en la educación musical. "Las comunidades de práctica musical son grupos sociales en los que los miembros están conectados a través de la música" y con ello se proporcionan condiciones específicas para una comunidad de práctica" (Westvall; Aragao 2019, p. 241-242).

De aquí se sigue que la visión de una práctica musical desde el colectivo, dará cuenta de un giro ontológico del sujeto individual al sujeto colectivo, cuya esencia es la de estar en concertación, en permanente relación intersubjetiva. Pese al desarrollo de la didáctica musical entre los siglos XVIII al XIX "al amparo de la expansión del modelo conservatorio y el desarrollo de la industria editorial, no ha habido desarrollo de una pedagogía del hacer musical colectivo" (Shifres; Gonnet, 2015, p. 58).

\section{Desarrollo Sostenible}

La concepción del desarrollo sostenible se sitúa como una necesidad imperiosa del hacer social desde cualquiera de sus ámbitos. Es un complejo asunto que implica cambios en lo individual, lo organizacional y lo social como un todo, requiriendo para ello un aprendizaje que promueva dicha transformación hacia la sustentabilidad (Palma; Ávila, 2016, p. 1-2). Un cambio paradigmático que ya no solamente aborda la relación del hombre con la naturaleza, sino que trae consigo una nueva concepción de dicha relación: la emergencia de un criterio, o visión integradora tendiente al fortalecimiento de relaciones colectivas y de cooperación, donde la contextualización de las prácticas educativas orientadas desde la complejidad sean capaces de "crear un ciclo real de acción- reflexión-acción relacionado con los retos socioambientales" (Jacobi, Ferraz de Toledo; Grandisoli; 2016, p. 2)

Desde la aparición del término 2030 (López; Ayala; Orellanes, 2017; Carro-Suárez; Sarmiento-Paredes; Rosano-Ortega, 2017; Zarta, 2018) han proliferado discursos desde todas las esferas socio-politicas, impulsadas principalmente por organismos multilaterales tales como la ONU y sus respectivas agencias, instituciones e instancias vinculadas a los estadosnaciones desde donde se articulan politicas direccionadas a lo sostenible, lo sustentable y lo sostenido, lo cual genera aristas que han dificultado precisar en ciertos momentos el concepto de sustentabilidad. Lo más conveniente es llamarlo sustentable porque "éste término, a más de su 
aspecto temporal, incluye conceptos que interrelacionan aristas muy importantes que preocupan a todos los seres de la tierra, guardando relación con el presente y futuro del planeta" (Zarta, 2018, p. 411-412).

Destacan por igual en el manejo discursivo de la sustentabilidad las dimensiones o ejes de la sustentabilidad, sin los cuales es imposible hablar de esta. Ya en la Carta de la Tierra, de acuerdo a lo reseñado por López; Ayala; Arellanes (2017) se advertía la necesidad de superar los limites que para el momento se tenía en torno al desarrollo sustentable, razón de que el mencionado documento fuese organizado en dieciséis (16) principios y a su vez agrupados en cuatro (4) apartados: respeto y cuidado de la vida, integridad ecológica, justicia social y económica y finalmente, democracia, no violencia y paz (p. 66). En el mismo orden de ideas, la sintesis de la sustentabilidad expresada en el conocido "Triángulo de Nijkamp", simboliza la relación entre sustentabilidad ambiental, equidad social y crecimiento económico (Zarta, 2018, p. 415).

Carro-Suárez; Sarmiento-Paredes; Rosano-Ortega (2017) citando a Ackhar (2005), toman en consideración el modelo planteado en base a "cuatro dimensiones de sustentabilidad: social, económica, ambiental e institucional" (p. 355). Palma; Ávila (2016) reconocen dos ejes: el socioeconómico y el ambiental. Establecen por tanto que: "el eje socioeconómico cubre el nivel de importancia dado al bienestar humano y la igualdad. El eje ambiental abarca la prioridad ambiental desde lo tecnocéntrico a lo eco-céntrico" (p. 3). Por igual, una noción importante que se conjuga dentro de la idea de desarrollo sostenible es el de biósfera (Aguirre, 2017) remitiendo con esto a la condición política y axiológica que implica la problemática de la sostenibilidad. "La sustentabilidad y la supervivencia de seres vivos innegablemente son necesarios en un sentido pragmático, pero no suficientes para dotar de sentido a la vida y las relaciones entre los vivientes" (p. 80).

De igual manera, el tema de las politicas públicas en torno a la sustentabilidad es reseñado por la bibliografia consultada como uno de los vacíos que aqueja el tratamiento del tema, lo cual vincula no sólo la estructura de los estados-naciones, sino que por igual atañe muy directamente a la educación desde la acción de planificación y ejecución de politicas-planes-estrategias y con ello fundamentalmente a las instituciones universitarias. "Los esfuerzos se han enfocado a la definición de políticas públicas o de especulaciones académicas que finalmente no se han concretado en transformaciones reales tanto de enfoques metodológicos, que den cuenta de la realidad aludida" (López-Tamayo 2015, p. 24). A juicio de Spraul; Thaler (2019) "el sector público ha gobernado principalmente hacia la sostenibilidad mediante la regulación de las cuestiones ecológicas y sociales procurando esfuerzos específicos de cada país para cambiar el comportamiento de las corporaciones" (p. 3-5). Es importante considerar en el mismo orden de ideas, las voces que emergen desde las comunidades 
indígenas o zonas rurales, donde se ensayan modelos signados como "buen vivir" o "modos de vida sustentables" que en muchos casos difieren del concepto de sustentabilidad (Galván; Fermán; Esejel, 2016), desde los cuales pueden surgir elementos de orden teórico y práctico que "abrirían nuevas perspectivas en los estudios sobre la sustentabilidad en comunidades indígenas" (p. 7).

\section{Educación ambiental}

El ámbito de la educación es también abordado desde la sostenibilidad (Luna-Nemecio, 2019a). A partir de la revisión documental se da cuenta de una educación para la sustentabilidad como "un excelente campo de conocimiento para ampliar la creciente capacidad de las entidades sociales en realizar tareas comunes relacionadas con iniciativas sostenibles" (Jacobi; Ferraz de Toledo; Grandisoli 2016, p. 3), lo cual involucra tanto los procesos como los resultados. Para los autores citados anteriormente, el aprendizaje social es la base epistemológica que orienta el planteamiento de una educación para sustentabilidad a partir de las aportaciones principalmente de autores como Bandura, reconociendo la acción recíproca entre el estudiante y el entorno para la formación de las conductas de aprendizaje. En este mismo sentido expresan Araújo; Lorenzo; Varela; Álvarez (2015) que la educación para la sustentabilidad "es un proceso intencional, sostenido en el tiempo, que implica la adquisición de conocimiento conceptuales y valores, así como el desarrollo de actitudes, aptitudes y modos de actuar en interacción social" (p. 41).

Schmitt; Anicet; Schutel (2016) reconocen que el planteamiento de la sustentabilidad, lo mismo que el desarrollo sostenible emergen como posibilidad de repensar y cuestionar el modelo de desarrollo de las naciones (p. 2), donde la educación juega un papel importante en el cambio de mentalidades. De esta manera, la educación para la sostenibilidad se centra en "formar estudiantes con los conocimientos, competencias y comprensión que se requiere para tomar decisiones en base a las implicaciones económicas y sociales del ambiente” (p. 2), teniendo por base el aprendizaje social en fundamentación de las diferentes prácticas educativas.

Desde 1972 se comienza hablar de Educación Ambiental a partir de la Conferencia Internacional sobre medio ambiente celebrada en Estocolmo donde se le concedió "la preponderancia para generar los cambios, mediante la adquisición de conocimientos, actitudes y valores, que permitan enfrentar seriamente la crisis ambiental" (Severiche-Sierra; Gómez-Bustamante; Jaimes-Morales, 2016, p. 269). Uno de los más aspectos más importantes a destacar en la concepción de la Educación Ambiental es el que emana del Congreso Iberoamericano de Educación Ambiental celebrado en México en 1992, donde se establece que "la educación ambiental es eminentemente politica y un instrumento muy importante para alcanzar la sostenibilidad en lo ambiental y en lo social" (Galindo, 2015, p. 8). De igual manera, al 
concebirse una educación ambiental que no se reduce a los problemas ecológicos sino a otras dimensiones, alinea el enfoque de cambio, de trascendencia de limites disciplinares, permitiendo de este modo nuevos niveles de realidad. "Los asuntos del ambiente son más que un problema ecológico, una crisis de pensamiento y comprensión, ontológica y epistemológica con la forma en la que la civilización occidental ha comprendido el ser (la existencia), los entes y las cosas" (ValderramaHernández; Alcántara; Limón 2017, p. 969).

Por su parte, y en el ámbito universitario, diversas perspectivas expresan sus diferencias, críticas y señalan aspectos necesarios de atender en torno a la educación ambiental tales como la presencia en el currículo, lo mismo que las distintas líneas de investigación hacia dónde dirige sus esfuerzos. En este sentido, encontramos observaciones en cuanto a la real contextualización de los contenidos de la educación ambiental y su pertinencia didáctica de cara a las realidades existentes (Severiche-Sierra; Gómez-Bustamante; Jaimes-Morales, 2016, p. 270). "Es necesario establecer lineamientos para poder superar la reacción inmediata y poco reflexionada que se genera por lo mediático de la crisis ambiental" (TovarGálvez, 2017, p. 534), para de esta manera fortalecer las estructuras y procesos en la gestión educativa de las universidades, permitiéndoles "reafirmar el protagonismo de las universidades en la formación de profesionales y de ciudadanos que tenga nuevas lecturas y acciones que signifiquen otras formas de relación y de existir en su medio socio-biofísico histórico" (p. 534). La educación ambiental "no se reduce a formar una ciudadanía para "conservar la naturaleza", "concienciar personas" o "cambiar conductas", se le asigna una tarea retadora: es educar para cambiar la sociedad" (González-Escobar, 2017, p. 6).

La transformación radical en la relación entre el individuo y su entorno, natural o construido, trae consigo una transformación de la sociedad en sí misma, "lo que lleva a comprender la complejidad de dichas relaciones y a permitir que los actores sociales intervengan en esta realidad de manera adecuada y justa"(p. 4). No se trata, como bien lo expresan Aguilar; Mercon; Silva (2017), de difundir concepciones de tipo ecológico, "sino de una propuesta educativa profundamente social y politica, crítica, reflexiva y propositiva" (p. 14). Se precisan entonces valoraciones de las estructuras axiológicas en sentido exploratorio-normativo desde donde se conjugan epistemológicamente formas de la realidad social (Costel, 2015, p. 381). De allí que, "el significado sociocultural debe relacionarse con la idea de la educación ambiental, dentro de la cual conceptos como la formación y el propósito tienen un papel importante" (p. 1381).

Cabe la pena destacar el planteamiento derivado de las corrientes críticas que inauguran horizontes epistemológicos para la configuración de una perspectiva más consustancial con la problemática ambiental. Torres (2015) nos presenta la propuesta de una educación para la compatibilidad, 
más que de una educación ambiental, la cual conecta al sujeto con las diferentes dimensiones del ambiente como lo son: la sociedad, la naturaleza, la humanidad y la cultura (p. 232-233). Así mismo la noción de ecosofía (Molina-Motos, 2018), busca integrar planteamientos en el orden de una pedagogía ecológica y una ecología social, "pensar y proponer alternativas educativas que abracen la aspiración compartida de todas las ecofilosofias de lograr la realización de la vida en el planeta, lo que también implica lógicamente la vida humana" (Molina-Motos, 2019, p. 8-9).

A partir del registro y análisis documental, resulta pertinente la articulación de una categoría desde la cual se pueda abordar una práctica educativa musical fundada en la acción de un colectivo en cuyo seno se suscite el acto de concertación como vivencia principalmente inter-subjetiva de reconocimiento de sí mismo a partir del relacionamiento con el otro mediado por la creación artístico-musical. Su acción pedagógica debe orientarse a propiciar una vivencia de creación conjunta, de trabajo colaborativo, de inter-subjetividad, tendiente a construir un ambiente de coexistencia para el manejo de los saberes esenciales que hacen posible lo poiético, y al propio tiempo, la formación integral del sujeto individual a partir del sujeto colectivo representado en la banda, la orquesta, el coro o un ensamble.

La dimensión social y cultural, en este sentido, son factores determinantes para la formulación de una educación musical que combina no sólo el desarrollo de las competencias necesarias para el desempeño sino que la práctica misma de la música da cuenta de condiciones que le son inherentes como hecho fundamentalmente politico, cuando se orienta a la participación de todos por igual, al garantizar su acceso principalmente a sectores vulnerables socialmente y emplea sus estrategias didácticas para la manifestación plena del sujeto a través del lenguaje artístico de los sonidos.

Educación Musical para el Desarrollo Sostenible (EMDS) abre alternativas desde la socioformación como via para atender la crisis ambiental cuando se comprende que la dimensión social y cultural del ser humano es un factor fundamental para revertir de los cambios negativos al modelo vigente de acción. Se dirige principalmente al fortalecimiento de la condición de sujeto social que vivencia tal condición ontológica al expresarse por medio del lenguaje artístico de los sonidos al propiciarse el acto de la concertación. Se ofrece como espacio inclusivo en el que tienen cabida todos por igual permitiendo el reconocimiento de la diversidad. Su enfoque en la formación por competencias se articula en función de la conjugación de los saberes necesarios orientados al desempeño con sentido crítico y atendiendo al criterio fundamental de la permanencia de la vida. 


\section{CONCLUSIONES}

La revisión documental realizada en torno a categorías como: educación musical, desarrollo sostenible y educación ambiental tendiente a develar el conjunto de conocimientos resultantes de la acción investigativa con miras a encontrar elementos de orden onto-epistémicos y metodológicos para orientar la formulación de una Educación Musical para el Desarrollo Sostenible (EMDS) desde la práctica colectiva de la música, permite establecer con suficiente claridad, que la conjugación de las tres categorías es pertinente.

La educación musical se ofrece como un campo abierto de enormes posibilidades para la generación de alternativas tendientes a contribuir con los necesarios equilibrios que se requieren para afrontar la problemática del desarrollo sostenible de nuestro tiempo actual a partir de planteamientos pedagógicos-didácticos que articulen fines y propósitos de la disciplina artístico-musical y el desarrollo sostenible (Luna-Nemecio; Tobón; JuárezHernández, 2019) en una acción educativa inclusiva, promotora de competencias para la vida. Por tanto, se considera viable concretar una propuesta formativa en educación musical que considere la práctica musical dentro de los criterios de la socioformación orientada hacia el desarrollo sostenible asumiendo plenamente la ontología desde el sujeto colectivo. La asunción de un nuevo sujeto para la educación musical orientada al desarrollo sostenible es elemento fundamental para redefinir los propósitos de una praxis educativa conjugando el orden disciplinar junto al orden socio cultural, con lo cual, la educación musical tanto en espacios formales y no formales se orienta a la vivencia desde lo colectivo, ya no desde el sujeto individual virtuoso sino desde la comunidad concertada.

En este sentido, la sostenibilidad, como criterio integrador y postura epistémica se abre paso en las distintas áreas del conocimiento para conducir acciones tendientes a un equilibrio de las diferentes dimensiones que son concomitantes en la necesidad de revertir las conductas y procederes que operan la lógica capitalista de esta modernidad continuada que ha erosionado las posibilidades de permanencia de la vida en el planeta. La educación orientada a la sostenibilidad surge como vehículo indispensable en la tarea transformadora que la crisis ambiental reclama. El ambiente ya no es el sitio exclusivo para nombrar el mundo natural, ni la relación del hombre con la naturaleza, no es ya un asunto exclusivamente de tipo ecológico o ambientalista, que se regula con acciones coyunturales sin el concierto de otros factores altamente vinculantes como el económico, el cultural, el social y el político. Hablar de una educación para el desarrollo sostenible tan sólo es posible desde la acción-reflexión-acción constante y permanente en atención al dinamismo del mundo actual. En el caso de la educación ambiental, las corrientes de pensamiento son concomitantes en torno a la imperiosa necesidad de trascender limites disciplinarios, fronteras 
onto-epistémicas y metodológicas en pos de nuevos cimientos para afrontar las graves consecuencias de un modelo de producción, entendido no sólo en términos económicos, sino por igual en la idea de generación de patrones de conducta y comportamiento que signan acciones contrarias a la vida.

\section{BIBLIOGRAFÍA}

AGUILAR, Maria., MERCON, J., SILVA, Evodia. Aportaciones de las percepciones socio-ecológicas a la Educación Ambiental. Entreciencias, México, v.5, n.15, p.14-24, 2017.

AGUIRRE, Jorge. La democracia líquida: exigencia de la sustentabilidad ecopolítica. Araucaria Revista Iberoamericana de Filosofía, Politica y Humanidades, España, v.19, n.38, p. 73-96, 2017.

ARAÚJO, Rocío; LORENZO, María Asunción; VARELA, Mercedes; ÁLVAREZ, Maria Mercedes. Educando en desarrollo sustentable: El índice de género y medio ambiente. Opción, Maracaibo, v.31, n.6, p. 36-54, 2015.

ASPRILLA, Ligia. Educar en la música: una aproximación crítica al talento y la educación musical. Aula, España, n.21, p. 63-83, 2015.

BATES, Vincent. Faith, Hope and Music Education. Action, Criticism and Theory for Music Education, v.17, n.2, p. 1-21, 2018.

BERMEO-YAFFAR, Faridy; HERNÁNDEZ-MOSQUEDA, José; TOBÓNTOBÓN, Sergio. Análisis documental de la V heurística mediante la cartografia conceptual. Ra Ximhai, v.12, n.6, p. 103-121, 2016.

CARRO-SUÁREZ, Jorge; SARMIENTO-PAREDES, Susana; ROSANOORTEGA, Genoveva. La cultura organización y su influencia en la sustentabilidad empresarial. La importancia de la cultura en la sustentabilidad empresarial. Estudios Generales, n.33, p. 352-365, 2017.

CIFE. Metodología del registro documental para la búsqueda y organización de la información científica. 1. ed. México: CIFE, 2016.

COSTEL, Esi. Didactic Options for the Environmental Education. Procedia Social and Behavioural Sciences, n.180, p. 1380-1385, 2015.

DUSSEL, Enrique. Filosofía de la liberación. México: Fondo de Cultura Económica. 2011.

FERNÁNDEZ, Ana María. Las lógicas colectivas. Imaginarios, cuerpos y multiplicidades. 2. ed. Buenos Aires: Biblos. 2008.

FERNÁNDEZ-JIMÉNEZ, Antonio; JORQUERA-JARAMILLO, María-Cecilia. El sentido de la educación musical en una educación concebida como motor de 
la economía del conocimiento: una propuesta de marco filosófico. Revista Complutense de Investigación en Educación Musical, Madrid, n.14, p. 95107, 2017.

FLORES-MONDRAGÓN, Gonzalo. Marx y Freire. Educación crítica y sustentable. Ecocience International Journal, v.1, n.1, p. 13-28, 2019.

GALINDO, Leticia. La educación ambiental en la virtualidad: un acercamiento al estado del arte. RIDE, México: v.5, n.10, p. 1-43, 2015.

GALVÁN, Danaé; FERMÁN, José; ESPEJEL, Ileana. ¿Sustentabilidad comunitaria indigena? Un modelo integral. Sociedad y Ambiente, Chiapas: v.4, n.11, p. 4-22, 2016.

GARCÍA, Narciso José López. Educación musical y currículo en la enseñanza primaria española: de la legislación general a la concreción autonómica. Revista da Abem, v. 26, n. 41, p. 56-76, 2018.

GOMES, Vania; MOTTA, Carolina. Environmental education in distance learning in Environmental Engineering at Federal University of Sao Carlos, Brazil; potentialities and limitations towards a critical techno-scientific education. Brazilian Journal of Science and Technology, v.3, n.7, p.1-14, 2016.

GONZÁLEZ-ESCOBAR, Carlos. La educación ambiental ante el problema ético del desarrollo. Revista Electrónica Educare, v.21, n.2, p. 296-314, 2017.

JACOBI, Pedro; FERRAZ DE TOLEDO, Renata; GRANDISOLI, Edson. Education, sustainability and social learning. Brazilian Journal of Science and Technology, v.3, n.3, pp1-8, 2016.

LÓPEZ, Rosalia; AYALA, Dante; ARELLANES, Yaayé. Gobernabilidad democrática y desarrollo local sustentable. Economia y sociedad. Morelia, v.21, n.36, p. 61-75, 2017.

LÓPEZ-TAMAYO, Nicolás. La ciudad para todos: gestión y sustentabilidad con sentido social. Revista Legado de Arquitectura y Diseño, México, n. 18, p. 23-34, 2015.

LUNA-NEMECIO, Josemanuel; TOBÓN, Sergio; JUÁREZ-HERNÁNDEZ, Luis. Socioforming and complexity: towards a new concept of sustainable social development. Human Development and Socioformation. v. 1, n.2, 2019.

LUNA-NEMECIO, Josemanuel. La importancia de la educación para lograr el desarrollo social sostenible. Ecocience International Journal, v. 1, n.1, p. 5$10,2019$.

- Megaproyectos. Acumulación de capital y sostenibilidad:

reconfiguración capitalista del territorio y devastación ambiental. Revista de Geografía Espacios, v.16, n.8, p. 1-9, 2018. 
MARTÍNEZ, Miguel. Ciencia y arte en la metodología cualitativa. México: Trillas, 2004.

MIEDIJENSKY, Shirley; ABRAMOVICH, Anat. Implementation of "Education for Sustainability" in three elementary schools- What can we learn about a change process? EUROASIA, v.15, n.10, pp1-18, 2018.

MEJÍA, María. Una educación ambiental desde la perspectiva cultural para la formación de profesores en ciencias naturales. Luna Azul, Caldas, n.43, p. 354-385, 2016.

MOLINA-MOTOS, David. Ecophilosophical Principles for an Ecocentric Environmental Education. Education Science, v.9, n.37, p. 1-15. 2019.

ORTEGA-CARBAJAL, Maria; HERNÁNDEZ-MOSQUEDA, José; TOBÓNTOBÓN, Sergio. Análisis documental de la gestión del conocimiento mediante la cartografia conceptual. Ra Ximhai, México, v.11, n.4, p. 141160, 2015.

PALMA, Lisiane; AVILA, Eugenio. Transformative learning to promote sustainability: inserting the third level of learning in management programs. Brazilian Journal of Science and Technology, v.3, n.9, p. 1-17, 2016.

PEÑALBA, Alicia. La defensa de la Educación Musical desde las neurociencias. Revista Complutense de Investigación en Educación Musical, Madrid, n.14, p. 109-127, 2017.

RAMÍREZ-HURTADO, Carmen. Aportaciones de la educación musical a la educación por la paz: buscando la transverdad en la era de la posverdad. Revista Electrónica Complutense de Investigación en Educación Musical, Madrid, n.14, p. 129.151, 2017.

REIS, João Gomes; DUARTE, Pedro. O currículo, a educação musical e as realidades individuais de cada estudante: um ensaio em defesa da inclusão cultural no ensino de música. Revista da Abem, v. 26, n. 41, p. 5-20, 2018.

SANTOYO-LEDESMA, Diana. Enfoque del Desarrollo Social Sostenible y la Gestión del Talento Humano en el contexto de la Socioformación. Ecocience International Journal, v. 1, n.1, p. 85-91, 2019.

SEVERICHE-SIERRA, Carlos; GÓMEZ-BUSTAMANTE, Edna; JAIMESMORALES, José. La educación ambiental como base cultural y estrategia para el desarrollo sostenible. Telos, Maracaibo, v.18, n.2, p. 226-281, 2016.

SHIFRES, Favio; GONNET, Daniel. Problematizando la herencia colonial en la educación musical. Epistemus, 3 (2), p. 51-67, 2015.

SICHERL, Bárbara; DENAC, Olga, JERNEJA, Konstanca. Analysis of Music Education objectives in learning domains. Procedia Social Behavioral Sciences, n.186, p. 95-104, 2015. 
SIERRA, Vicente. La Educación Musical en el Proyecto Roma. Revista Interuniversitaria de Formación del Profesorado, v.92, n.32.2, p. 97-106, 2018.

SPRAUL, Katharina; THALER, Julia. Partnering for good? An Analysis of how to achieve sustainability-related outcomes in public-private partnership. Business Research, p. 1-27, 2019.

TOBÓN, Sergio. La socioformación: avances y retos en la sociedad del conocimiento. En: COMIE (Ed.). Conferencias magistrales del Congreso Nacional de Investigación Educativa. México: COMIE, p. 179.234, 2015.

TORRES, Guillermo. La pedagogía ambiental: hacia un nuevo paradigma educativo. Entreciencias: diálogos en la Sociedad del Conocimiento, México, v.3, n.7, p. 227-240, 2015.

TOURIÑAN, José; LONGUEIRA, Silvana. La música como ámbito de educación. Educación "por" la música y educación "para” la música. Teoría de la Educación, v.22, n.2, p. 151-181, 2010.

TOVAR-GÁLVEZ, Julio. Pedagogía ambiental y didáctica ambiental: tendencia en la educación superior. Revista Brasileira de Educación, v.22, n.69, p. 519-538, 2017.

VALDERRAMA-HERNÁNDEZ, Rocío; ALCÁNTARA, L; LIMÓN, D. The complexity of environmental education: teaching ideas and strategies from teachers. Procedia Social and Behavioural Science, n.237, p. 968-974, 2017.

VARAS, Ibar. Teoría dialógica de la educación. Barquisimeto, Venezuela: Ediciones Al Sur. 2008.

WESTVALL, Maria; ARAGAO, Pedro. Communities of Musical Practice: Transformative contexts for intercultural communication. El oído pensante, v.7, n.1, p. 236-250, 2019.

ZARTA, P. La sustentabilidad o sostenibilidad: un concepto poderoso para la humanidad. Tabula Rasa, Colombia, n.28, p. 409-423, 2018. 
Oswaldo Antonio Rodrígez Reinoso es docente e investigador del Conservatorio "Vicente Emilio Sojo", Fundación Compañía de Ópera de Occidente, Sistema de Orquestas y Coros Juveniles e Infantiles de Venezuela, Fundación Coro de Manos Blancas, en áreas como técnica vocal, práctica coral, canto lírico e investigación musical. Fundador de Centro de Investigación y Documentación del Conservatorio "Vicente Emilio Sojo" (BarquisimetoVenezuela), conferencista en diversos congresos, seminarios y eventos académicos nacionales e internacionales con varias publicaciones en revistas indizadas. Tenor barquisimetano, formado en el Conservatorio de Música "Vicente Emilio Sojo" de Barquisimeto, Venezuela, por el Maestro Angelo D'Addona completando formación con: Mirella Freni, Suso Mariategui, Edelmiro Arnaltes, Aquiles Machado, Giuliana Panza, Patrizia Morandini, Lotti Ipinza, Isabel Palacios y Lucy Ferrero. Su carrera artística comprende iniciada en 2001 incluye la ópera, la zarzuela, el recital y el sinfónico coral. Roles primarios y co-primarios en autores como: Mozart, Handel, Puccini, Giménez, Luzuriaga, Ruiz, Ochoa y Guerrero. Solista invitado de diversas orquestas en países como Venezuela, Colombia, Cuba, Nicaragua, España, Perú, Ecuador y Honduras. https://orcid.org/0000-0002-4894-7186

Josemanuel Luna-Nemecio es Doctor en Geografía por la Universidad Nacional Autónoma de México. Maestro en Ciencias Sociales por la Universidad Autónoma del Estado de Morelos. Licenciado en Economía por la Universidad Nacional Autónoma de México. Actualmente es Profesor Investigador del Centro Universitario CIFE, en la ciudad de Cuernavaca, México. Es Director Editorial de las revistas Forhum. International Journal of Social Sciences and Humanities y de Ecocience. International Journal. Se ha dedicado a impartir cátedra en diversas Universidades públicas y privadas del país tanto a nivel licenciatura y posgrado. Ha participado como ponente en diversos Foros, Congresos, Coloquios, Conferencias y Talleres a nivel nacional e internacional. Sus principales líneas de investigación son: desarrollo social sostenible, crítica de la economía política, producción social del espacio, ambiente, cambio climático y geografía política de los recursos hídricos. Sus últimas publicaciones son: Calentamiento Global y conflictividad sociohídrica ante el colapso ambiental del estado de Morelos, México y Megaprojects, capital acummulation and sustainability: the capitalist reconfiguration of the territory and the environmental devastation. https://orcid.org/0000-0002-6850-3443 